\title{
Food preferences and weight change during low-fat and low- carbohydrate diets
}

\author{
Megan A. McVay a, b, *, Corrine I. Voils ${ }^{\text {b, c }}$, Paula J. Geiselman ${ }^{\text {d, e }}$, Valerie A. Smith ${ }^{\text {b, f }}$, \\ Cynthia J. Coffman ${ }^{\text {b, f, }}$, Stephanie Mayer ${ }^{\mathrm{g}}$, William S. Yancy Jr. ${ }^{\text {b, c }}$ \\ a Division of Behavioral Medicine, Department of Psychiatry and Behavioral Sciences, Duke University Medical Center, Durham, NC, United States \\ ${ }^{\mathrm{b}}$ Center for Health Services Research in Primary Care, Durham VA Medical Center, Durham, NC, United States \\ ${ }^{c}$ Division of General Internal Medicine, Department of Medicine, Duke University Medical Center, Durham, NC, United States \\ d Department of Psychology, Louisiana State University, Baton Rouge, LA, United States \\ e Pennington Biomedical Research Center, Louisiana State University, Baton Rouge, LA, United States \\ ${ }^{\mathrm{f}}$ Department of Biostatistics \& Bioinformatics, Duke University, Durham, NC, United States \\ ${ }^{g}$ Division of Endocrinology and Metabolism, Virginia Commonwealth University, Richmond, VA, United States
}

\section{A R T I C L E I N F O}

\section{Article history:}

Received 9 February 2016

Received in revised form

19 April 2016

Accepted 27 April 2016

Available online 29 April 2016

Keywords:

Food preferences

Low-fat diet

Low-carbohydrate diet

\begin{abstract}
A B S T R A C T
Understanding associations between food preferences and weight loss during various effective diets could inform efforts to personalize dietary recommendations and provide insight into weight loss mechanisms. We conducted a secondary analysis of data from a clinical trial in which participants were randomized to either a 'choice' arm, in which they were allowed to select between a low-fat diet $(\mathrm{n}=44)$ or low-carbohydrate diet $(\mathrm{n}=61)$, or to a 'no choice' arm, in which they were randomly assigned to a low-fat diet $(n=49)$ or low-carbohydrate diet $(n=53)$. All participants were provided 48 weeks of lifestyle counseling. Food preferences were measured at baseline and every 12 weeks thereafter with the Geiselman Food Preference Questionnaire. Participants were 73\% male and 51\% African American, with a mean age of 55. Baseline food preferences, including congruency of food preferences with diet, were not associated with weight outcomes. In the low-fat diet group, no associations were found between changes in food preferences and weight over time. In the low-carbohydrate diet group, increased preference for low-carbohydrate diet congruent foods from baseline to 12 weeks was associated with weight loss from 12 to 24 weeks. Additionally, weight loss from baseline to 12 weeks was associated with increased preference for low-carbohydrate diet congruent foods from 12 to 24 weeks. Results suggest that basing selection of low-carbohydrate diet or low-fat diet on food preferences is unlikely to influence weight loss. Congruency of food preferences and weight loss may influence each other early during a lowcarbohydrate diet but not low-fat diet, possibly due to different features of these diets.

Clinical trial registry: NCT01152359.
\end{abstract}

(c) 2016 Elsevier Ltd. All rights reserved.

\section{Introduction}

A low-fat/low-calorie diet has been the primary weight loss approach advocated by public health organizations for the past several decades (Marantz, Bird, \& Alderman, 2008). However, with increasing evidence that low-carbohydrate diets are as effective as low-fat diets (Foster et al., 2003; Gardner et al., 2007; Sacks et al. 2009; Shai et al. 2008), low-carbohydrate diets are growing in

\footnotetext{
* Corresponding author. Duke University Medical Center, Department of Psychiatry and Behavioral Science, Box 3862, Durham, NC, 27710, United States.

E-mail address: megan.mcvay@duke.edu (M.A. McVay).
}

acceptability. Thus, individuals have the option to select among these dietary approaches as well as others, such as the Mediterranean diet. However, it is unclear which factors should guide their selection. Identifying factors that differentiate who will be more successful on which diet regimen may facilitate personalized selection of dietary approach.

One factor that may differentiate which individuals are likely to be more successful with one dietary approach over another is food preferences. Because food preferences influence food selection (Furst, Connors, Bisogni, Sobal, \& Falk, 1996; Glanz, Basil, Maibach, Goldberg, \& Snyder, 1998), it can be hypothesized that assignment to a diet that is more aligned with one's food preferences will result 
in greater dietary adherence, and therefore potentially, weight loss. Alternatively, congruency between food preferences and diet could hinder weight loss, as individuals may be more likely to overconsume preferred foods if those foods are allowable on their diet.

Some of the most commonly preferred foods in the US are not congruent with either the low-fat or low-carbohydrate diet (Drewnowski, Kurth, Holden-Wiltse, \& Saari, 1992; Yang, Buys, Judd, Gower, \& Locher, 2013). These foods are high in both fat and sugar, such as cakes, ice cream, and candy, or are high in both fat and complex carbohydrates, such as French fries and macaroni and cheese. It is currently unknown whether individuals with strong preferences for these highly preferred food types will be more successful with a low-fat or a low-carbohydrate diet. Information about the relationship between food preferences and weight loss during different diets could facilitate personalized recommendations of dietary strategies.

While baseline food preferences may influence weight loss, there is also evidence that food preferences may change over the course of a weight loss attempt (Ledikwe et al. 2007; Martin et al., 2011). In particular, researchers have found that preferences for foods that are not compatible with a diet decrease during that diet (Ledikwe et al. 2007; Martin et al., 2011). Less well understood is whether these changes in food preferences may influence weight outcomes, such that individuals who experience greater change in preference also experience greater weight loss. We are aware of only one study that has examined associations between changes in food preference and body weight (Martin et al., 2011), and no association was found. However, low-fat and low-carbohydrate diets appear to have been combined in those analyses; given that the relationship between baseline food preferences and food intake may differ as a function of diet types, combining these two diets in one analyses may have masked any effects of food preference on weight loss.

To test relationships among food preferences, weight loss diet regimen, and weight loss, we performed secondary analyses of data from a doubly randomized clinical trial. Participants in this trial were randomized to either a 'choice' arm, in which they were allowed to select between a low-fat or low-carbohydrate diet, or to a 'no choice' arm, in which they were randomly assigned to a lowfat or low-carbohydrate diet. They completed a 48-week behavioral weight loss intervention. In that study, we found that individuals who selected their own diet and those who were randomly assigned a diet did not differ in weight loss (Yancy et al. 2015). In the current paper, we analyzed a validated measure of food preferences administered at baseline and every 12 weeks thereafter, allowing us to directly test longitudinal associations between food preferences and weight.

We had two primary aims related to the relationship between food preferences, dietary regimen (low-fat or low-carbohydrate), and weight loss. The first aim was to determine if baseline food preferences are associated with weight loss in low-fat and lowcarbohydrate diets at the end of a 48-week weight loss intervention. Food preferences examined included high-fat/high-sugar food preferences, high-fat/high-carbohydrate food preferences, and a measure of congruency of food preferences with dietary approach. We focused specifically on high-fat/high-sugar food preferences and high-fat/high-carbohydrate food preferences because they are highly preferred foods and because they are incompatible with both low-fat and low-carbohydrate diets. The results of this aim will provide evidence to address the question of whether assigning individuals to a diet on the basis of their food preferences is likely to improve outcomes. We conducted Aim 1 analyses exclusively in the 'no choice' condition in order to take advantage of randomization, which ensures that there is not confounding due to self-selection of diet that had occurred in the diet choice condition. This is particularly important in this study given that diet selection in the diet choice condition was associated with dietary preferences (McVay et al., 2014).

Our second aim was to examine if changes in food preferences are associated with changes in weight. For these analyses, the lowfat and low-carbohydrate diets were examined separately given the possibility that greater congruency with a given diet may lead to greater weight loss. We combined the choice and no choice condition for these analyses in order to have adequate power; although concerns about confounding exist in these analyses, the risk is reduced due to adjusting for covariates in multivariable models.

\section{Methods}

\subsection{Participants}

Details of the study design, procedures and intervention have been reported (Yancy et al. 2015). Study participants were recruited from the Durham Veterans Affairs (VA) Medical Center between June 2011 and June 2012 via advertisements posted in clinic and hospital areas and referrals by VA health care personnel. Additionally, a search of electronic health records for patients meeting study criteria was conducted, with potentially eligible patients sent a recruitment letter with a telephone number to contact if they were interested.

Patients were eligible for the study if they had BMI $\geq 30 \mathrm{~kg} / \mathrm{m}^{2}$, were in stable health, reported a desire to lose weight, agreed to attend regular visits per study protocol, had access to a telephone and reliable transportation, and had a VA provider. Exclusion criteria were age over 75; certain chronic or unstable medical or psychiatric conditions that might interfere with weight loss or make weight loss unsafe (e.g. kidney or liver disease; daily insulin use; dementia); concurrent enrollment in a conflicting study; use of weight loss therapy in the previous month; any previous enrollment in a weight loss research study using a low-fat/low-calorie or low-carbohydrate diet. We also excluded females who were pregnant, breastfeeding, or at risk of becoming pregnant during the course of the study (i.e., not using birth control). Eligibility was determined during screening telephone calls and at an in-person screening visit, during which all participants provided written informed consent and completed a measure of food preferences, the Geiselman Food Preference Questionnaire (FPQ; described in detail in Methods Section, below). All study procedures were approved by the Durham VA Institutional Review Board.

\subsection{Procedures}

\subsubsection{Randomization and diet initiation}

After the screening visit, participants were randomized to the diet choice arm or the no choice arm. Following randomization, participants in the diet choice arm attended an informed decisionmaking group session to guide them through the diet choice process. Participants in this arm received FPQ results in print form indicating which diet most closely aligned with their food preferences, but they were also told that they should ultimately choose on their own which diet to follow, based on other information provided in the session or any additional information they might gather in the ensuing week. In the session, participants were then provided with an overview of the two diets (foods emphasized, foods to be avoided, sample menus, etc.), and were informed that they would be provided the option to switch diets 12 weeks into the intervention. At this meeting, diet choice arm participants were able to ask questions but were asked to refrain from stating opinions about the diets and from discussing diet choice with other group participants so as to avoid influencing one another. One week 
following the informed decision making session, participants were contacted via telephone by the study dietitian to answer questions and solicit their diet choice. Then, participants were scheduled in their first group weight loss session for the following week, either for the low-fat diet or low-carbohydrate diet. Factors associated with diet selection have been reported, which included food preferences as well as other factors (e.g., expected health benefits; McVay et al., 2014).

Participants randomized to the no choice arm underwent a second randomization to either the low-fat diet or lowcarbohydrate diet. At the first group session, these participants received an overview of the study procedures and were advised not to start their diet until their next group session 2 weeks later in order to parallel the timeline of the diet choice arm.

\subsubsection{Dietary intervention and option to switch diets}

Group weight loss sessions were held every two weeks for the first 24 weeks of the study. Over the subsequent 24 weeks of the study, participants met monthly and had interposed individual monthly telephone calls from the dietician focused on goal setting. Each small group contained approximately 15 participants in the same arm and on the same diet. All sessions were led by a registered dietician with experience delivering both low-fat/low-calorie diets and low-carbohydrate diets.

The low-fat diet prescribed total fat intake of less than $30 \%$ of daily intake, saturated fat intake of less than $10 \%$, and cholesterol intake of less than $300 \mathrm{mg}$ per day throughout the study. Low-fat/ low-calorie participants were given a caloric goal of 500 less than their estimated maintenance energy requirement and provided guidance on how to meet that goal. Caloric restriction could be further reduced or increased for individuals based on their progress towards weight loss goals. The low-carbohydrate diet prescribed an initial total carbohydrate intake of $20 \mathrm{~g}$ or less per day without calorie restriction. Carbohydrate intake could be increased gradually as participants' weight loss goals were approached or if cravings threatened adherence. Further information on the prescribed diets used in this study is provided in Table 1.

At week 12, diet choice arm participants met individually with study personnel to indicate whether they would like to switch to the other diet. Participants who chose to switch diets participated in an individual counseling session to obtain information on the new diet and thereafter switched to group sessions for that diet and arm.

\subsection{Measures}

\subsubsection{Baseline demographic and clinical measurements}

Participants reported their age, sex, race, and if they had received a diabetes diagnosis (yes or no). Trained research assistants blinded to study arm and diet measured participants' body weights on a standardized digital scale (Tanita, Inc.) with participants wearing light clothes and shoes removed. Weight was measured at baseline and at all group visits and outcome assessments; for purposes of these analyses, we used weight measurements from outcome assessments at weeks $0,12,24,36$, and 48 .

\subsubsection{Food preferences}

The Geiselman FPQ was used to obtain hedonic ratings of foods with specific macronutrient contents and to calculate macronutrient preferences (Geiselman et al., 1998). The FPQ was developed as a 2 (Fat: High fat and Low fat) X 3 (Other macronutrient: High complex carbohydrate, High simple sugar, and High protein) assessment, yielding the following six cells indicative of food type preference: high-fat/high-complex carbohydrate, high-fat/highsugar, high-fat/high-protein, low-fat/high-complex carbohydrate, low-fat/high-sugar, and low-fat/high-protein. Twelve foods are included in each of the six cells of the FPQ for a total of 72 foods, which are listed in random order. The FPQ asks participants to rate how much they like each of the 72 foods on a 9-point Likert-type scale $(1=$ dislike extremely; $5=$ neutral, neither like nor dislike; $9=$ like extremely). Foods listed on the FPQ vary significantly and systematically in macronutrient content. Each food item in each of the three high-fat cells has $\geq 45 \%$ fat (expressed as percentage of the total kilocalories in a given food), and each food in each of the three low-fat cells has $<20 \%$ fat. Other macronutrient criteria for the FPQ are: high complex carbohydrate ( $\geq 30 \%$ complex carbohydrate); high sugar ( $\geq 30 \%$ sugar); and high protein ( $\geq 13 \%$ protein, although most items were $20-35 \%$ protein). The foods included in the FPQ are common sources of macronutrients in the typical U.S. diet and/ or are among the favorite foods of specific populations, including men and women with obesity, and Whites and African-Americans (Geiselman et al., 1998). Example foods include pudding, pot roast, French fries, ice cream, and several different egg preparations. The FPQ has demonstrated test-retest reliability and validity, and construct validity has been supported by correlations with food intake measured in a laboratory setting and with self-reported dietary intake (Geiselman et al., 1998). FPQs were completed by both study arms at screening ("week 0") and weeks 12, 24, 36, and 48.

\subsection{Analyses}

\subsubsection{Aim 1: baseline food preferences and weight loss outcomes}

For Aim 1, we used two of the FPQ subscales: high-fat/high sugar and high-fat/high-carbohydrate preferences. We also constructed a variable from other FPQ subscales to describe participants' relative preference for low-carbohydrate diet vs low-fat diet foods. This relative diet congruency preference variable was created by subtracting FPQ low-fat/high-complex carbohydrate preference subscale from high-fat/high-protein preference subscale. These two FPQ subscales were used because they differentiate with regard to congruency with the diets studied; the other four FPQ subscales are either similarly congruent with both diets (i.e., low-fat/highprotein) or are similarly incongruent with either diet (i.e., highfat/high-sugar, high-fat/high-complex carbohydrate, and low-fat/ high-sugar). Positive scores on the relative diet congruency preference variable reflect greater congruency of preference with lowcarbohydrate diet (the higher the score, the more congruent), and negative scores reflect greater congruency with low-fat diet (the lower the score, the more congruent). We used relative congruency rather than a measure of absolute congruency for these analyses because it mimics how one might go about using food preferences to select between a low-fat diet and low-carbohydrate diet; that is, one would focus on those preferences that are acceptable for one diet type and not for the other, rather than on preferences for foods that are acceptable on both or neither diet type.

To determine if baseline food preferences were associated with weight loss during a low-fat or low-carbohydrate diet, we conducted a linear regression analysis with the dependent variable of weight change at 48 weeks and the independent variables of food preference, diet type, and the interaction of food preference and diet type. Missing 48 week weight measurements were estimated using best linear unbiased predictors from the mixed models used for primary analysis of weight outcomes (Littell, Milliken, Stroup, Wolfinger, \& Schabenberger, 2006; Yancy et al. 2015). As previously noted, this analysis was conducted only in the no-choice study arm to take advantage of randomization to diet that occurred in that arm, eliminating the possibility of confounding due to self-selection of diet. Separate regression analyses were conducted to test each of the three baseline food preference variables of interest (high-fat/high-sugar food preferences, high-fat/ 
Table 1

Description of low-fat and low-carbohydrate diets prescribed in current study.

\begin{tabular}{|c|c|c|}
\hline & Low-fat diet & Low-carbohydrate diet \\
\hline $\begin{array}{l}\text { Fat and cholesterol } \\
\text { recommendation }\end{array}$ & $\begin{array}{l}\text { Total fat: } \leq 30 \% \text { of daily intake; saturated fat } \leq 10 \% \text { of daily intake; } \\
\text { cholesterol } \leq 300 \mathrm{mg} / \text { day. }\end{array}$ & No limitations \\
\hline $\begin{array}{l}\text { Carbohydrate } \\
\text { recommendation }\end{array}$ & $\begin{array}{l}\text { No specific limitations, but encouraged to consume less refined sugar and } \\
\text { more fiber. }\end{array}$ & $\leq 20 \mathrm{~g} /$ day initially \\
\hline $\begin{array}{l}\text { Protein } \\
\quad \text { recommendation }\end{array}$ & No limitations & No limitations \\
\hline $\begin{array}{l}\text { Calorie } \\
\text { recommendation }\end{array}$ & $500 \mathrm{kcal}$ less than their estimated maintenance energy requirement & No limitations \\
\hline $\begin{array}{l}\text { Other } \\
\text { recommendations }\end{array}$ & $\begin{array}{l}\text { Caloric restriction recommendations could be increased or decreased based } \\
\text { on individual's progress towards weight loss goals. }\end{array}$ & $\begin{array}{l}\text { Carbohydrate intake could be increased gradually as participants' } \\
\text { weight loss goals were approached or if cravings threatened adherence. }\end{array}$ \\
\hline
\end{tabular}

high-carbohydrate, and relative diet congruency).

Of particular interest in these analyses was the interaction effect between food preference and dietary approach. A significant interaction, or moderation, effect would indicate that individuals with a given food preference have better weight loss outcomes on one diet than the other. For example, a significant positive interaction between relative diet congruency preference and diet would indicate that preference for low-carbohydrate diet foods contributed to greater weight loss in the low-carbohydrate diet group compared to the low-fat diet group, and thus that congruency between food preferences and diet are beneficial for weight loss.

\subsubsection{Aim 2: longitudinal changes in food preferences and weight}

For Aim 2, we again used the high-fat/high-sugar and high-fat/ high-carbohydrate preference scores from the FPQ. We also developed absolute congruency preference variables for both the low-fat and low-carbohydrate diet. Low fat-absolute congruency preference is a measure of congruency of food preferences with the low-fat diet, and was constructed as the average of low-fat/highprotein and low-fat/high-complex carbohydrate preference scores. Low-carbohydrate absolute congruency preference is a measure of congruency of food preferences with the low-carbohydrate diet, and was constructed as the average of low-fat/high-protein and high-fat/high-protein preference scores. Higher scores reflect greater congruency with the low-fat or low-carbohydrate diet, respectively. These absolute preference variables were developed for Aim 2 rather than using relative diet congruency variable from Aim 1 because, for this aim, we were interested in changes in the totality of foods that are congruent with each diet rather than those that are uniquely congruent with each diet.

To examine longitudinal changes in food preferences, we conducted linear mixed models with each preference score as the dependent variable and time as the independent variable. Diet regimens were examined separately and study arms (choice and no choice) were combined. For these analyses, separate regressions were conducted for each of the four food preferences variables of interest (high-fat/high-sugar, high-fat/high-complex carbohydrate, low-fat absolute congruency preference, and low-carbohydrate absolute congruency preference)

Next, to determine if changes in food preferences were prospectively associated with change in weight during a low-fat or low-carbohydrate diet, an autoregressive, cross-lagged structural model was estimated using maximum likelihood in Mplus software (see Fig. 1). For these models, we were interested in congruency of food preferences as it relates to weight change, so we fit two separate unique models: one examining changes in weight and low-fat diet absolute congruency preferences in the low-fat diet group, and one examining changes in weight and low-carbohydrate absolute congruency preferences in the low-carbohydrate diet group (choice and no choice arms combined). We tested a panel model with 5 waves (baseline, 12 weeks, 24 weeks, 36 weeks, and 48 weeks), with each wave containing weight and food preference (see Fig. 1). Covariates in these models included choice condition, age, sex, diabetes status and smoking status. Model fit was assessed with $\chi 2$ test, root mean square error of approximation (RMSEA; Steiger \& Lind, 1980), comparative fit index (CFI; Bentler, 1990), and Tucker-Lewis Index (TLI; Tucker \& Lewis, 1973). Conventional cutoffs for acceptable fit are RMSEA $\leq 0.06$, CFI $\geq 0.95$ and TLI $\geq 0.95$ (Hu \& Bentler, 1999). Of note, 5 participants in the Choice arm switched diets when given the option at 12 weeks ( 3 in lowcarbohydrate diet group, 2 in low-fat). Given the small number of participants that switched diets, all participants were analyzed within the study to which diet they were either originally randomized or originally chose. For all analyses, a p-value of $<0.05$ was considered statistically significant.

\section{Results}

\subsection{Descriptive characteristics}

Study participants included 105 participants randomized to the diet choice condition (61 low-carbohydrate diet, and 44 LFD) and 102 to the no choice condition (53 low-carbohydrate diet, and 49 LFD). In the choice condition, 87 (82.9\%) participants completed the final weight assessment at 48 weeks. In the no-choice condition, 88 (86.4\%) participants completed the 48 week weight assessment. The majority of patients who did not complete the assessment were lost to follow-up. See the primary outcomes paper for additional details on participant retention (Yancy et al., 2015). The average age of participants was 54.7 years old, and $73 \%$ were male. Participants were 51\% African American and 45\% Caucasian. Twenty-three percent self-reported a diagnosis of diabetes. Mean baseline FPQ scores are provided in Table 2 .

\subsection{Aim 1: baseline food preferences and weight loss outcomes}

Regression analyses testing associations between baseline food preferences and 48 week weight loss (in the no choice condition only) showed no significant main effects or interaction effects for any of the three food preference variables examined (Table 3). These results indicate that baseline preferences for high-fat/highsugar and high-fat/high-complex carbohydrate foods were not predictive of weight outcomes nor did weight change differ between the two diets examined based on preference for these foods. Additionally, the absence of an interaction effect between diet type and relative diet congruency preference indicates that congruency of preferences with diet did not affect weight loss.

\subsection{Aim 2: longitudinal associations of change in food preferences and weight}

Table 4 provides results from linear mixed model analyses 


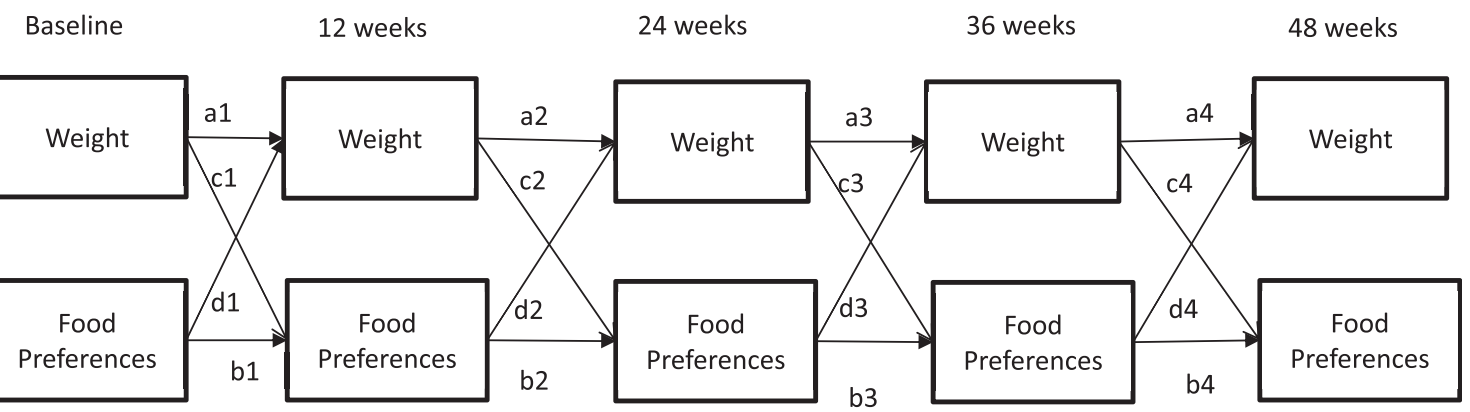

Fig. 1. Autoregressive cross-lagged model of change in weight and food preferences.

testing changes in food preferences over time. Among low-fat diet participants, preference decreased from baseline to follow-up time points (weeks 12, 24, 36, 48) for all four types of food preferences examined (high-fat/high-sugar, high-fat/high-complex carbohydrate, low-fat absolute congruency preference, low-carbohydrate absolute congruency preference). Among low-carbohydrate diet participants, preference decreased from baseline to follow-up points for three of the four preferences examined: high fat/high sugar, high fat/high complex carbohydrate, and low-fat absolute congruency preferences. In contrast, there was not significant change in preference for low-carbohydrate absolute congruency foods at any of the follow-up periods examined.

Results of the autoregressive models, presented in Table 5, demonstrate the relationship between food preferences and weight change over time. In the low-fat diet group, model fit was marginal, and no prospective associations were found between food preferences examined and weight. In the low-carbohydrate diet group, model fit was acceptable. Growth in low-carbohydrate absolute congruency preferences from baseline to 12 weeks was associated with greater weight loss between 12 and 24 weeks ( $\beta=-0.05$, $\mathrm{p}=0.008$ ). Greater weight loss between baseline and 12 weeks was associated with greater increase in low-carbohydrate absolute congruency preferences by 24 weeks ( $\beta=-0.14, \mathrm{p}=0.04$; Table 5 ).

\section{Discussion}

Identifying factors that distinguish between those who are likely to be more successful with one dietary regimen than another is critical for guiding personalized treatment recommendations and has potential to improve weight loss outcomes. Food preferences are an obvious target for personalized selection of dietary approaches. In the current study, we assessed whether food preferences, including congruency of preference with diet, would distinguish whether an individual was more likely to succeed on either a low-fat diet or a low-carbohydrate diet, and thus guide personalized recommendations. Our results did not support the hypothesis that individuals would lose more weight when prescribed a diet that has greater congruency with their food preferences. We also examined associations between changes in food preferences and weight change during the interventions. We found that an increase in preference for dietcongruent foods during the first 12 weeks of the intervention was associated with later weight loss in the low-carbohydrate diet group. Furthermore, weight loss during the first 12 weeks of the intervention was associated with a subsequent increase in preference for diet-congruent foods. In the low-fat group, no longitudinal associations were found between food preferences and weight loss.

These results suggest that selecting a diet that is consistent with one's food preferences may not be beneficial or detrimental. These results are consistent with studies showing that people do not lose more weight, and may actually lose less weight, when they receive a preferred or self-selected diet (Borradaile et al. 2012; Yancy et al. 2015). However, those past studies did not look specifically at the role of food preferences, which are only one of several factors that influenced diet preference/selection (McVay et al. 2014). Our results suggest that it is reasonable for individuals select diet based on their food preferences, but without the expectation that doing so will facilitate greater weight loss.

High-fat/high-sugar foods and high-fat/high-complex carbohydrate foods are restricted in both low-fat or low-carbohydrate diets, yet they are often identified as among the most preferred foods among Americans (Drewnowski et al. 1992; Geiselman et al. 1998). Furthermore, there has been some suggestion in popular media that a low-carbohydrate diet may be particularly effective for individuals who are especially drawn to sugar (Eenfeldt, 2014). Contrary to this notion, we found that degree of preference for high-fat/high-sugar foods or high-fat/high-complex carbohydrate foods was not associated with likelihood to succeed on either diet examined. The overall lack of influence of baseline dietary preferences on weight change indicates a need to identify factors other than dietary preferences that may differentially affect success across different diets if personalized recommendations are going to be beneficial. Researchers should examine other potential factors

Table 2

Baseline FPQ variable values among all study participants.

\begin{tabular}{|c|c|c|c|}
\hline Food preference variables ${ }^{\mathrm{a}}$ & Mean (SD) & Range-LL & Range-UL \\
\hline Relative diet congruency preference $(n=204)^{b}$ & $1.2(1.3)$ & -2.8 & 5.1 \\
\hline High-fat/high-sugar preference $(\mathrm{n}=205)$ & $6.2(1.6)$ & 1.4 & 9.0 \\
\hline High-fat/high-complex carbohydrate preference $(\mathrm{n}=202)$ & $5.9(1.3)$ & 2.0 & 8.7 \\
\hline Low-fat diet absolute congruency preferences $(n=205)$ & $6.1(1.1)$ & 2.8 & 9.0 \\
\hline Low-carbohydrate diet absolute congruency preferences $(n=206)$ & $6.7(1.1)$ & 3.8 & 9.0 \\
\hline
\end{tabular}

Notes.

a The range of possible values for relative preference congruency is -9 to 9 and for other variables is $0-9$.

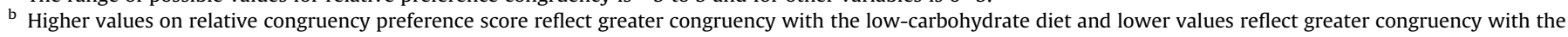
low-fat diet. 
Table 3

Linear regression results predicting 48-week weight change among 'no choice' study arm participants ( $\mathrm{n}=102)$.

\begin{tabular}{|c|c|c|c|}
\hline & Overall model $\mathrm{R}^{2}$ & Standardized coefficient & $\mathrm{p}$-Value ${ }^{\mathrm{a}}$ \\
\hline Relative congruency preference model & 0.02 & & 0.51 \\
\hline Relative congruency preference & & -0.043 & 0.16 \\
\hline Diet type & & -0.014 & 0.34 \\
\hline Relative congruency preference $\times$ diet type & & 0.35 & 0.26 \\
\hline High fat/high sugar preference model & 0.01 & & 0.74 \\
\hline High fat/high sugar preference & & -0.32 & 0.30 \\
\hline Diet type & & -0.45 & 0.31 \\
\hline High fat/high sugar preference $\times$ diet type & & 0.60 & 0.27 \\
\hline High fat/high complex carbohydrate preference model & 0.03 & & 0.37 \\
\hline High fat/high complex carbohydrate preference & & -0.44 & 0.16 \\
\hline Diet type & & -0.85 & 0.10 \\
\hline High fat/high complex carbohydrate preference $\times$ diet type & & 1.00 & 0.10 \\
\hline
\end{tabular}

a P-value tests significance of overall model or of the coefficient for individual variable in the model.

Table 4

Model-estimated food preference values over time among all participants.

\begin{tabular}{|c|c|c|c|c|c|c|c|c|}
\hline & $\begin{array}{l}\text { High fat/high } \\
\text { sugar, M (SE) }\end{array}$ & $\begin{array}{l}\text { p- } \\
\text { Value }^{\mathrm{a}}\end{array}$ & $\begin{array}{l}\text { High fat/high complex } \\
\text { carbohydrate, M (SE) }\end{array}$ & $\begin{array}{l}\text { p- } \\
\text { Value }^{\mathrm{a}}\end{array}$ & $\begin{array}{l}\text { Absolute congruency preference- } \\
\text { low fat diet, } \mathrm{M}(\mathrm{SE})\end{array}$ & $\begin{array}{l}\text { p- } \\
\text { Value }^{\mathrm{a}}\end{array}$ & $\begin{array}{l}\text { Absolute congruency preference-low } \\
\text { carbohydrate diet, M (SE) }\end{array}$ & $\begin{array}{l}\text { p- } \\
\text { Value }^{\mathrm{a}}\end{array}$ \\
\hline \multicolumn{9}{|c|}{ Low-fat diet $(n=93)$} \\
\hline $\begin{array}{c}\text { Week } \\
0\end{array}$ & $6.09(0.18)$ & & $5.79(0.15)$ & & $6.04(0.14)$ & & $6.57(0.11)$ & \\
\hline $\begin{array}{c}\text { Week } \\
12\end{array}$ & $5.67(0.20)$ & 0.01 & $5.11(0.18)$ & $<0.0001$ & $5.79(0.16)$ & 0.08 & $6.20(0.13)$ & 0.003 \\
\hline $\begin{array}{c}\text { Week } \\
24\end{array}$ & $5.59(0.21)$ & 0.001 & $4.96(0.17)$ & $<0.0001$ & $5.65(0.16)$ & 0.002 & $6.11(0.14)$ & $<0.0001$ \\
\hline $\begin{array}{c}\text { Week } \\
36\end{array}$ & $5.52(0.21)$ & 0.003 & $4.93(0.17)$ & $<0.0001$ & $5.71(0.16)$ & 0.02 & $6.24(0.13)$ & 0.004 \\
\hline $\begin{array}{c}\text { Week } \\
48\end{array}$ & $5.59(0.20)$ & 0.006 & $4.96(0.17)$ & $<0.0001$ & $5.74(0.15)$ & 0.02 & $6.18(0.13)$ & 0.0007 \\
\hline \multicolumn{9}{|c|}{ Low-carbohydrate diet $(\mathrm{n}=112)$} \\
\hline $\begin{array}{c}\text { Week } \\
0\end{array}$ & $6.30(0.14)$ & & $6.08(0.12)$ & & $6.07(0.10)$ & & $6.74(0.10)$ & \\
\hline $\begin{array}{c}\text { Week } \\
12\end{array}$ & $5.90(0.17)$ & 0.004 & $5.43(0.15)$ & $<0.0001$ & $5.61(0.12)$ & $<0.0001$ & $6.66(0.10)$ & 0.30 \\
\hline $\begin{array}{c}\text { Week } \\
24\end{array}$ & $5.53(0.18)$ & $<0.0001$ & $5.11(0.16)$ & $<0.0001$ & $5.42(0.11)$ & $<0.0001$ & $6.64(0.11)$ & 0.26 \\
\hline $\begin{array}{c}\text { Week } \\
36\end{array}$ & $5.46(0.19)$ & $<0.0001$ & $4.98(0.16)$ & $<0.0001$ & $5.41(0.12)$ & $<0.0001$ & $6.64(0.11)$ & 0.28 \\
\hline $\begin{array}{c}\text { Week } \\
48\end{array}$ & $5.48(0.19)$ & $<0.0001$ & $5.07(0.16)$ & $<0.0001$ & $5.44(0.11)$ & $<0.0001$ & $6.62(0.10)$ & 0.23 \\
\hline
\end{tabular}

Notes. Values are among all study participants (Choice and No Choice) with available study data.

a P-value is for comparison of week 0 to subsequent week.

Table 5

Estimates from the mediator models for dietary outcomes.

\begin{tabular}{|c|c|c|c|c|c|c|c|c|c|c|c|c|c|c|c|c|}
\hline & & \multicolumn{8}{|c|}{ Low-carbohydrate diet $(\mathrm{n}=112)$} & \multicolumn{7}{|c|}{ Low-fat diet $(n=93)$} \\
\hline & & \multicolumn{3}{|c|}{$\begin{array}{l}\text { Low-carbohydrate diet } \\
\text { congruency preference }\end{array}$} & & \multicolumn{3}{|c|}{$\begin{array}{l}\text { Low-fat diet congruency } \\
\text { preference }\end{array}$} & & \multicolumn{3}{|c|}{$\begin{array}{l}\text { Low-carbohydrate diet } \\
\text { congruency preference }\end{array}$} & & \multicolumn{3}{|c|}{$\begin{array}{l}\text { Low-fat diet congruency } \\
\text { preference }\end{array}$} \\
\hline & & Beta $^{a}$ & SE & p-Value & & Beta $^{a}$ & SE & p-Value & & Beta $^{a}$ & SE & p-Value & & Beta $^{a}$ & SE & p-Value \\
\hline \multicolumn{17}{|c|}{ Weight $\rightarrow$ food preference } \\
\hline Weeks $0 \rightarrow 12(\mathrm{c} 1)$ & & 0.099 & 0.09 & 0.24 & & 0.027 & 0.09 & 0.78 & & -0.062 & 0.10 & 0.53 & & -0.042 & 0.10 & 0.69 \\
\hline Weeks $12 \rightarrow 24(\mathrm{c} 2)$ & & -0.139 & 0.07 & 0.04 & & -0.05 & 0.08 & 0.50 & & 0.150 & 0.08 & 0.07 & & 0.108 & 0.08 & 0.19 \\
\hline Weeks $24 \rightarrow 36$ (c3) & & -0.077 & 0.07 & 0.29 & & -0.07 & 0.08 & 0.38 & & -0.115 & 0.08 & 0.16 & & -0.135 & 0.08 & 0.10 \\
\hline Weeks $36 \rightarrow 48$ (c4) & & 0.007 & 0.09 & 0.94 & & 0.003 & 0.09 & 0.97 & & -0.066 & 0.08 & 0.40 & & -0.071 & 0.08 & 0.37 \\
\hline \multicolumn{17}{|c|}{ Food preferences $\rightarrow$ weight } \\
\hline Weeks $0 \rightarrow 12(\mathrm{~d} 1)$ & & -0.006 & 0.02 & 0.77 & & -0.007 & 0.02 & 0.74 & & -0.016 & 0.02 & 0.39 & & -0.006 & 0.02 & 0.78 \\
\hline Weeks $12 \rightarrow 24(\mathrm{~d} 2)$ & & -0.048 & 0.02 & 0.008 & & -0.03 & 0.02 & 0.10 & & -0.024 & 0.02 & 0.25 & & -0.01 & 0.02 & 0.63 \\
\hline Weeks $24 \rightarrow 36$ (d3) & & 0.005 & 0.02 & 0.798 & & -0.009 & 0.02 & 0.64 & & -0.005 & 0.02 & 0.75 & & 0.007 & 0.02 & 0.67 \\
\hline Weeks $36 \rightarrow 48(\mathrm{~d} 4)$ & & 0.000 & 0.02 & 0.99 & & 0.014 & 0.02 & 0.42 & & -0.011 & 0.02 & 0.52 & & -0.009 & 0.02 & 0.59 \\
\hline \multicolumn{17}{|l|}{ Model fit indices } \\
\hline $\mathrm{X} 2$ & 139.5 & & & & 123.1 & & & & 136.8 & & & & 150.7 & & & \\
\hline RMSEA & 0.10 & & & & 0.08 & & & & 0.10 & & & & 0.113 & & & \\
\hline CFI & 0.96 & & & & 0.96 & & & & 0.95 & & & & 0.94 & & & \\
\hline TLI & 0.94 & & & & 0.95 & & & & 0.94 & & & & 0.92 & & & \\
\hline
\end{tabular}

Note.

a All coefficient values are standardized, b. Degrees of freedom for all chi-squared analyses for model fit is 69 . 
that may be used to personalize dietary recommendations, such as metabolic or genetic profile, cost of foods, ease of availability of certain types of food over others, or prior diet experience.

We observed a reduction in preference for foods that are highfat/high-sugar and high-fat/high-carbohydrate over the course of both a low-fat and low-carbohydrate diet. This is consistent with previous findings showing a decrease in preference for foods that are incongruent with a dietary regimen (Ledikwe et al. 2007; Martin et al. 2011) and suggests that one means by which low-fat and low-carbohydrate diets may affect weight is by reducing preferences for foods that are inconsistent with those diets. One possible explanation for this change is the psychological phenomena of cognitive dissonance. Cognitive dissonance occurs when an attitude (e.g., preference for high-fat/high-sugar foods) and a behavior (e.g., reduction in consumption of high-fat/high-sugar foods) are in conflict. A drive to reduce dissonance results in change in attitude (e.g., reduced preference for high-fat/high-sugar foods; Festinger, 1962). It is also possible that food preferences change because dietary changes contribute to neurobiological changes in brain regions that control food preferences and reward centers (Berthoud, 2012).

While both diets led to decreased preference for high-fat/highsugar and high-fat/high-carbohydrate foods, a difference was found between the low-fat and low-carbohydrate diets in regard to diet-congruent foods. Those who were on a low-fat diet reported a decrease in preferences for low-fat diet congruent foods, whereas those who were on the low-carbohydrate diet did not report a significant change in preferences for low-carbohydrate diet congruent foods. The reasons for this difference between diets are unclear, however, among our study participants, lowcarbohydrate diet foods tended to be more highly preferred than low-fat congruent foods at baseline (McVay et al. 2014). To the extent that these foods are more preferred, they may also retain their preference to a higher degree than low-fat diet congruent foods.

Our study is the first, to our knowledge, to show that the development of greater congruency of food preferences with diet is associated with greater weight loss in the low-carbohydrate diet. Notably, this is found only during the early stages of a weight loss effort, which is the time period when low carbohydrate diets tend to produce greater weight loss than low-fat diets. This suggests that one explanation for why some individuals lose more weight than others on a low-carbohydrate diet is because their preferences for foods change in a manner that is consistent with the diet. Further, this relationship appeared to be reciprocal; change in weight was also associated with subsequent change in food preference. It is unclear why these relationships are observed with a lowcarbohydrate diet but not the low-fat diet. One possibility is that the low-carbohydrate diet has more severe restrictions on macronutrients than the low-fat diet, and thus changes in macronutrient preference may be more crucial for success in this diet than in the low-fat diet.

This study has several limitations. First, the study was not powered specifically for these secondary analyses. Second, our study was conducted in a VA sample, which limits generalizability, and more research is needed to confirm these findings in diverse populations. Third, we studied only two dietary regimens; future studies might examine food preferences and weight change across other diet types. Restrained eating was not measured in this study and we cannot determine if it influenced our results. Finally, the model fit for our autoregressive models was marginal. Strengths of this study include use of validated measure of food preferences, longitudinal measuring of food preferences, and a participant sample rich in men and African Americans, individuals who are understudied in weight loss literature.

\section{Conclusions}

The results suggest that individuals and practitioners guiding individuals may select between diets on the basis of food preference, but that they should not expect that to influence weight loss. Future research may determine if monitoring changes in food preferences over the course of a low-carbohydrate diet and adjusting an intervention accordingly improves weight loss.

\section{Sources of support}

This research was supported by VA HSR\&D (IIR 09-381). Dr. Megan McVay is supported by a career development award from the National Heart, Lung, \& Blood Institute (K23 HL127334). Dr. Stephanie Mayer was supported by an NIH T32 grant: 5T32DK007012 Endocrinology and Metabolism Research Training Program. Dr. Corrine Voils was supported by a VA Research Career Scientist award (14-443).

\section{References}

Bentler, P. M. (1990). Comparative fit indexes in structural models. Psychological Bulletin, 107(2), 238.

Berthoud, H. R. (2012). The neurobiology of food intake in an obesogenic environment. Proceedings of the Nutrition Society, 71(04), 478-487.

Borradaile, K. E., Halpern, S. D., Wyatt, H. R., Klein, S., Hill, J. O., Bailer, B., et al. (2012). Relationship between treatment preference and weight loss in the context of a randomized controlled trial. Obesity, 20(6), 1218-1222.

Drewnowski, A., Kurth, C., Holden-Wiltse, J., \& Saari, J. (1992). Food preferences in human obesity: carbohydrates versus fats. Appetite, 18(3), 207-221.

Eenfeldt, A. (2014). The sugar cravings are gone now. Retrieved from http://www. dietdoctor.com/sugar-cravings-gone-now.

Festinger, L. (1962). A theory of cognitive dissonance (Vol. 2). Stanford University Press.

Foster, G. D., Wyatt, H. R., Hill, J. O., McGuckin, B. G., Brill, C., Mohammed, B. S., et al. (2003). A randomized trial of a low-carbohydrate diet for obesity. New England Journal of Medicine, 348(21), 2082-2090.

Furst, T., Connors, M., Bisogni, C. A., Sobal, J., \& Falk, L. W. (1996). Food choice: a conceptual model of the process. Appetite, 26(3), 247-266.

Gardner, C. D., Kiazand, A., Alhassan, S., Kim, S., Stafford, R. S., Balise, R. R., et al. (2007). Comparison of the Atkins, Zone, Ornish, and LEARN diets for change in weight and related risk factors among overweight premenopausal women: the A TO Z Weight Loss Study: a randomized trial. Jama, 297(9), 969-977.

Geiselman, P. J., Anderson, A. M., Dowdy, M. L., West, D. B., Redmann, S. M., \& Smith, S. R. (1998). Reliability and validity of a macronutrient self-selection paradigm and a food preference questionnaire. Physiology \& Behavior, 63(5), 919-928.

Glanz, K., Basil, M., Maibach, E., Goldberg, J., \& Snyder, D. A. N. (1998). Why Americans eat what they do: taste, nutrition, cost, convenience, and weight control concerns as influences on food consumption. Journal of the American Dietetic Association, 98(10), 1118-1126.

Hu, L. T., \& Bentler, P. M. (1999). Cutoff criteria for fit indexes in covariance structure analysis: conventional criteria versus new alternatives. Structural Equation Modeling: A multidisciplinary journal, 6(1), 1-55.

Ledikwe, J. H., Ello-Martin, J., Pelkman, C. L., Birch, L. L., Mannino, M. L., \& Rolls, B. J. (2007). A reliable, valid questionnaire indicates that preference for dietary fat declines when following a reduced-fat diet. Appetite, 49(1), 74-83.

Littell, R., Milliken, G., Stroup, W. W., Wolfinger, R. D., \& Schabenberger, O. (2006). SAS for mixed models (2nd ed.). Cary, NC: SAS Institute Inc.

Marantz, P. R., Bird, E. D., \& Alderman, M. H. (2008). A call for higher standards of evidence for dietary guidelines. American Journal of Preventive Medicine, 34(3), 234-240.

Martin, C. K., Rosenbaum, D., Han, H., Geiselman, P. J., Wyatt, H. R., Hill, J. O., et al. (2011). Change in food cravings, food preferences, and appetite during a lowcarbohydrate and low-fat diet. Obesity, 19(10), 1963-1970.

McVay, M. A., Voils, C. I., Coffman, C. J., Geiselman, P. J., Kolotkin, R. L., Mayer, S. B., et al. (2014). Factors associated with choice of a low-fat or low-carbohydrate diet during a behavioral weight loss intervention. Appetite, 83, 117-124.

Sacks, F. M., Bray, G. A., Carey, V. J., Smith, S. R., Ryan, D. H., Anton, S. D., et al. (2009), Comparison of weight-loss diets with different compositions of fat, protein, and carbohydrates. New England Journal of Medicine, 360(9), 859-873.

Shai, I., Schwarzfuchs, D., Henkin, Y., Shahar, D. R., Witkow, S., Greenberg, I., et al. (2008). Weight loss with a low-carbohydrate, Mediterranean, or low-fat diet. New England Journal of Medicine, 359(3), 229-241.

Steiger, J. H., \& Lind, J. C. (1980). Statistically based tests for the number of common factors. In Paper presented at the annual meeting of the Psychometric Society (Iowa City, IA). 
Tucker, L. R., \& Lewis, C. (1973). A reliability coefficient for maximum likelihood factor analysis. Psychometrika, 38(1), 1-10.

Yancy, W. S., Mayer, S. B., Coffman, C. J., Smith, V. A., Kolotkin, R. L., Geiselman, P. J., et al. (2015). Effect of allowing choice of diet on weight loss: a randomized trial.
Annals of Internal Medicine, 162(12), 805-814.

Yang, Y., Buys, D. R., Judd, S. E., Gower, B. A., \& Locher, J. L. (2013). Favorite foods of older adults living in the Black Belt Region of the United States. Influences of ethnicity, gender, and education. Appetite, 63, 18-23. 